\title{
Comorbidities Affecting Children with Epilepsy - Not a Novel Entity but New Insights Could Improve Holistic Care
}

\author{
Tandokazi Quvile and Jo M Wilmshurst
}

Department of Paediatrics and Child Health, Red Cross War Memorial Children's Hospital, University of Cape Town, South Africa

DOI: http://doi.org/10.17925/ENR.2016.11.01.16

\begin{abstract}
Children with epilepsy commonly suffer additional and significant neurological co-morbidities. Epilepsy syndromes can be associated with specific cognitive and behavioural phenotypes which can aid diagnosis. Early recognition and intervention of both epilepsy and the associated comorbidities can lead to improved neurocognitive outcomes. Intrinsic connectivity networks are influenced by multiple modalities including the stage of brain maturation in early onset epilepsies and epileptiform activity with resultant impact on neurocognitive function.
\end{abstract}

\section{Keywords}

Epilepsy, children, comorbidities, neurocognition, behaviour, neuropsychiatric, genetics, intrinsic connectivity networks

\begin{abstract}
Disclosure: Tandokazi Quvile and Jo Wilmshurst have nothing to disclose in relation to this article. This article is a short opinion piece and has not been submitted to external peer reviewers. No funding was received in the publication of this article. open Access: This article is published under the Creative Commons Attribution Noncommercial License, which permits any non-commercial use, distribution, adaptation and reproduction provided the original author(s) and source are given appropriate credit. Received: 20 March 2016 Published Online: 20 April 2016 Citation: European Neurological Review, 2016;11(1):16-7 Correspondence: Jo M Wilmshust, Paediatric Neurology Department, Department of Paediatrics and Child Health, Red Cross War Memorial Children's Hospital, Klipfontein Rd, Cape Town, South Africa 7700. E: Jo.wilmshurst@uct.ac.za
\end{abstract}

Children with epilepsy rarely suffer the condition in isolation. Cognitive, behavioural and psychiatric issues, which manifest as attention deficit hyperactivity disorder (ADHD), intellectual disability (ID), autism spectrum disorder (ASD), depression, unstable mood, and suicidality, are reported in $11-40 \%$ of those affected. ${ }^{1-4}$ These diverse comorbidities, which complicate the care and outcome of children, are often as much of a management challenge as the epilepsy itself, leading to significant health resource utilisation. ${ }^{5}$ This is compounded by multiple influences, including the underlying aetiology for the epilepsy, the baseline health of the child, socioeconomic status, access to care, the age of onset of the events, the frequency of seizures, the antiepileptic drugs used, and genetic make-up. Whilst there is awareness of these comorbidities, this does not always translate into clinical practice. The concept that people with epilepsy need multidisciplinary care is novel for many services which focus on control of seizures. ${ }^{3}$ Intervention for associated comorbidities is more likely to be reactive and opportunities for early intervention or prevention of exacerbation of symptoms lacking. ${ }^{4}$ Limited resources and capacity are additional compounding factors.

Behavioural phenotypes, however, can be useful through supporting epilepsy diagnoses, for example aggression and impulsivity in children with ring chromosome 20 aberration, rigid personalities and autistic traits in girls with PCDH19 mutations, and severe ID and Rett syndromelike features in girls with CDKL5 mutations. ${ }^{11}$ This is helpful in resource poor settings where access to genetic studies is limited. Closure of syndromic diagnoses influences care and counseling.

The World Health Organization Mental Health gap guideline includes epilepsy. ${ }^{6}$ Whilst contentious due to concern that it further stigmatizes the disease, there is no doubt that recognising and acknowledging the associated cognitive, behavioural and psychiatric deficits early on in a child's life, leads to better holistic care for the child. ${ }^{4}$

There are situations were early intervention can positively impact and reduce disease burden. West syndrome, a disorder consisting of epileptic spasms, developmental delay and hypsarrhythmia, carries a worse cognitive outcome the longer the lead-time to treatment intervention. ${ }^{10}$

The combined disorders of epilepsy, ASD and ID are not infrequent and this is especially so for children with epileptic encephalopathy. ${ }^{7,8}$ Early recognition and therapy targeting social deficits in children with epilepsy and ASD may result in improved cognitive and developmental outcomes. ${ }^{8}$ Treating epilepsy and even interictal discharges can also result in improved cognitive profiles in affected children. ${ }^{7}$ Up to 400 genes are linked to ASD, 50\% of which are involved in synaptic plasticity, which impacts on brain connectivity. A number of these ASD associated genes influence the balance of gamma-aminobutyric acid (GABA) and glutamate, both of which are important in the pathogenesis of epilepsy. ${ }^{9}$

Progress in the field of genetics has expanded understandings of various conditions, increasing knowledge of disease pathogeneses, how genetic mutations influence the clinical expression in the child, as well as the epilepsy semiology and the other comorbidities of the disease. ${ }^{9,12,13}$ Tuberous sclerosis complex is a good example of this. Understanding the role of mammalian target of rapamycin (mTOR) in the expression of the disease has led to targeted therapies and evidence that these interventions benefit disease expression across multiple areas from tumour growth to epilepsy control and even to 
neurocognition. ${ }^{14,15}$ Early and appropriate targeted therapy for affected children enables optimal outcomes in both seizure control but also cognitive outcome.

Understanding the influencers for overall brain function and subsequent expression is important to direct further research and subsequent care. ${ }^{16}$ Intrinsic connectivity networks (ICNs) reflect the organisation of functional networks at rest and in turn on neurocognitive development. Studies investigating ICNs have illustrated complex, dynamic and interwoven processes that evolve with brain maturation and are affected by both underlying aetiology, genetics, seizure frequency, interictal discharges and antiepileptic drugs. ${ }^{17-19}$ From the onset of childhood epilepsy, multiple domains of impaired cognition, abnormalities in brain structure and or connectivity are evident. ${ }^{18}$ Children with benign childhood epilepsy with centrotemporal spikes (BECTS) have transient or persisting cognitive and behavioural problems, even after seizure remission. Connectivity between bilateral homotropic voxels was found to have disrupted functional cooperation between hemispheres in these children. ${ }^{20}$ The hyposynchrony between the bilateral middle frontal gyrus was hypothesised to be involved in the decreased intelligence quotients of children with BECTS. ${ }^{20}$ Children with absence seizures had generalized-spike-and-wave discharge related functional excitation in the thalamus, as well as extensive inhibitions in other ICNs corresponding to higher-order cognitive processes and sparing of motor and perceptive processes. ${ }^{19}$ Further, early-onset epilepsy may have a deleterious impact on key white matter connections. ${ }^{18}$ There is evidence for both aberrant brain structure and cognitive delay being present early in the course of epilepsy which supports a neurodevelopmental contribution to the cognitive comorbidities of children with epilepsy. ${ }^{18}$ Transitory cognitive impairment (TCI) is reported in 50\% of individuals with epileptiform discharges, despite this the effects of interictal discharges on the neurocognitive development of children with medically refractory epilepsy is poorly understood. ${ }^{17}$ Interictal discharges may have a negative effect on the brain's ICNs. Large-scale network changes precede and follow interictal epileptiform discharges, the resilience of network topologies to interictal discharges is associated with stronger resting-state network connectivity, and vulnerability to interictal discharges is associated with worse neurocognitive outcomes. Based on this data it could be considered clinically important to suppress discharges in order to enhance more typical brain network development especially in children with focal epilepsy. ${ }^{17}$

Antiepileptic drugs (AEDS) can also influence neurobehavioural function in children. ${ }^{21}$ AEDs approved before the 1938 FDA act did not complete substantive human trial safety data analysis, these include phenobarbitone (registered in 1912) and phenytoin (registered in 1938). Agents released after 1993 received the most rigorous clinical trial evaluation. For these later products, the wealth of side effects is well documented. AEDs with GABAergic effects have the most important psychiatric and behavioural side effects. Influences on brain connectivity related to AED use have also been studied. ${ }^{22}$ The effects in ICNs related to early and chronic use of phenobarbitone would be of interest. This agent is reported to be associated with significant cognitive and behavioural adverse effects, as well as concern of influences on brain maturation. In many parts of the world for those children who are recognised to have epilepsy, and attain treatment, they are often dependent of limited supply of agents such as phenobarbitone and are unlikely to have associated comorbidities recognised or managed.

The wealth of knowledge in relation to this field only serves to emphasise the importance of providing early and appropriate intervention for these children on a world-wide scale. The comorbidities affecting children with epilepsy, whilst not a novel concept, may be remedial to the outcomes from new understandings which could lead to better care which should be transitioned across healthcare levels and regions in the future.
1. Terra VC de Paola L Silvado CE, Are children affected by epileptic neuropsychiatric comorbidities?, Epilepsy Behav, 2014;38:8-12

Austin JK, Perkins SM, Johnson CS, et al., Behavior problems in children at time of first recognized seizure and changes over the following 3 years, Epilepsy Behav, 2011;21:373-81.

3. Reilly C, Atkinson P, Das KB, et al., Neurobehavioral comorbidities in children with active epilepsy: a populationbased study, Pediatrics, 2014;133:e1586-93.

4. Guilfoyle SM, Wagner JL, Smith G, Modi AC, Early screening and identification of psychological comorbidities in pediatric epilepsy is necessary, Epilepsy Behav, 2012;25:495-500.

5. Puka K, Smith ML, Moineddin R, et al., Health resource utilization varies by comorbidities in children with epilepsy, Epilepsy Behav, 2016;57(Pt A):151-4.

6. World Health Organisation: Mental Health Gap Action Program mhGAP Intervention Guide for mental, neurological and substance use disorders in non-specialized health and substance use disorders in non-specialized health settings. 2009. Available at: www.who.int/mental/hed
evidence/mhGAP/en/(accessed 11 April 2016).

7. Jeste SS, Tuchman R, Autism Spectrum Disorder and Epilepsy: Two Sides of the Same Coin?, I Child Neurol, 2015;30:1963-71.

8. Tuchman R, Autism and Cognition Within Epilepsy: Social Matters, Epilepsy Curr, 2015;15:202-5.
9. Brooks-Kayal A, Epilepsy and autism spectrum disorders: are there common developmental mechanisms? Brain Dev 2010;32:731-8.

10. O'Callaghan FJ, Lux AL, Darke K, et al., The effect of lead time to treatment and of age of onset on developmental outcom at 4 years in infantile spasms: evidence from the United Kingdom Infantile Spasms Study, Epilepsia, 2011;52:1359-64

11. Wilmshurst JM, Berg AT, Lagae L, et al., The challenges and innovations for therapy in children with epilepsy, Nat Rev Neurol, 2014;10:249-60.

12. Sawyer NT, Helvig AW, Makinson CD, et al., Scn1a dysfunction alters behavior but not the effect of stress on seizure response, Genes Brain Behav, 2016:15:335-47.

13. McTague A, Howell KB, Cross JH, wt al., The genetic landscape of the epileptic encephalopathies of infancy and childhood, of the epileptic encephalopath,

14. Curatolo P, Aronica E, Jansen A, et al., Early onset epileptic encephalopathy or genetically determined encephalopathy with early onset epilepsy? Lessons learned from TSC, Eur J Paediatr Neurol, 2016;20:203-11.

15. Krueger DA, Wilfong AA, Holland-Bouley K, et al., Everolimus treatment of refractory epilepsy in tuberous sclerosis complex, Ann Neurol, 2013;74:679-87.
16. Hansen EC Battaglia D, Spiegler A et al., Functiona connectivity dynamics: modeling the switching behavior of the resting state, Neuroimage, 2015;105:525-35.

17. Ibrahim GM, Cassel D, Morgan BR, et al., Resilience of developing brain networks to interictal epileptiform discharges is associated with cognitive outcome, Brain, 2014;137(Pt 10):2690-702.

18. Addis L, Lin JJ, Pal DK, et al., Imaging and genetics of language and cognition in pediatric epilepsy, Epilepsy Behav, 2013:26:303-12.

19. Zhang Z, Liao W, Wang Z, et al., Epileptic discharges specifically affect intrinsic connectivity networks during absence seizures, J Neurol Sci, 2014:336:138-45.

20. WU Y, Ji GJ, Li K, et al., Interhemispheric Connectivity in DrugNaive Benign Childhood Epilepsy With Centrotemporal Spikes: Combining Function and Diffusion MRI. Medicine (Baltimore), 2015;94:e1550.

21. Glauser TA, Behavioral and psychiatric adverse events associated with antiepileptic drugs commonly used in pediatric patients, J Child Neurol, 2004;19 (Suppl 1):S25-38.

22. Haneef Z, Levin HS, Chiang S, Brain Graph Topology Changes Associated with Anti-Epileptic Drug Use, Brain Connect, 2015;5:284-91. 